\title{
New agreement announced
}

Align Technology B.V (part of Align

Technology, Inc. - NADSAQ: ALGN) and

Bupa Dental Care have announced a new agreement, which will increase the offer of the Invisalign system and iTero intraoral scanners across Bupa's network of dental practices in the UK \& Ireland, benefiting both patients and dentists alike.

Beginning with immediate effect, the new contract will also see Align provide dedicated education and training programmes for Bupa's network of dentists and orthodontists, so that they can leverage the latest innovations with confidence while providing patients with the highest possible quality care. With over 470 practices across the UK and Ireland, as well as ambitious growth plans, Bupa Dental Care is one of the market's biggest dental providers, carrying out over six million appointments each year.

Align Technology is a global medical device company focused on the design and manufacture of the Invisalign, the most advanced clear aligner system in the world. It produces more than 380,000 unique aligner parts per day, each one digitally designed to gradually straighten a patient's teeth. The Invisalign system nearly invisible appearance makes it a popular alternative to traditional braces.

For more information please visit www.invisalign.co.uk and www. bupadentalcare.co.uk.

\section{Combat tooth loss}

Dental implants have become incredibly popular as an effective treatment modality for partial or full edentulism, particularly among elderly patients. TBR's Z1 implant system can provide optimal dental function and aesthetics similar to that of a natural tooth, thanks to proven technology.

Combining a highly biocompatible titanium body with a unique zirconia collar, the $\mathrm{Z} 1$ promotes effective osseointegration, and encourages the soft tissue to heal around the implant in a way that mimics natural gingival growth. This reduces the risk of iatrogenic inflammation or infection, ensuring a highly satisfactory result.

For more information on the $\mathrm{Z} 1$ implant, visit tbr.dental, email hello@ denkauk.com or call 08007076212.

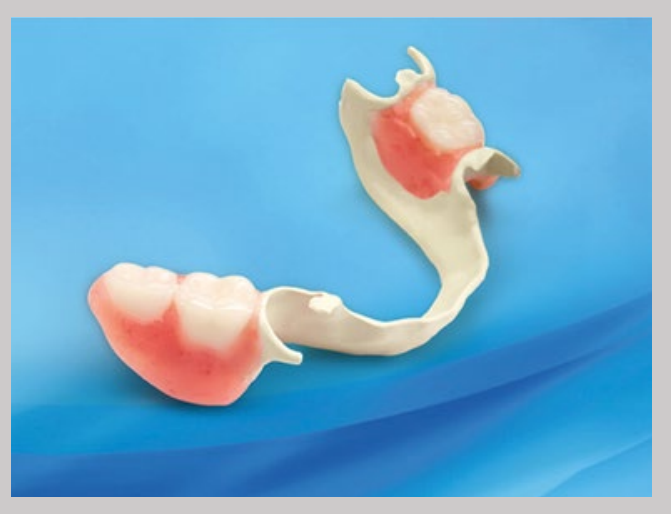

Unique social networking site for dental professionals launches

A unique online platform created to facilitate networking between dental professionals and patients has arrived in the form of OralHealthNet.

Registration is free, enabling OralHealthNet members - both individuals and clinics - to post a photo, contact details of their practice and their educational background. Further facilities are available for a small fee and right now the site is running a special deal, offering incredible value.

For anyone who signs up before the end of June 2019, a one-off payment of $£ 50$ will cover them until 1 January 2020 ,essentially offering four months free during the roll-out of the website as it is introduced to consumers

Ordinarily, the cost will be $£ 25$ per month to add treatments, services, prices, more photos, a social media news feed and a website link, as well as a way to make direct contact without exposing personal details, or $£ 50$ for the added extra of a live booking facility and to add videos.

This is your chance to showcase your talents and educate consumers about your services within this dedicated community and building stronger relationships.

For further information, visitoralhealthnet.co.uk or emailinfo@ oralhealthnet.co.uk.

Here has been an advertisement. 